\title{
Clinical practice guidelines
}

Editor's note: In the context of the Implementation Forum, we are interested in the application of clinical practice guidelines. To that end, we include this introduction to the topic of clinical practice guidelines.

This article describes the development, implementation, and appraisal of clinical practice guidelines. It also explores ways of increasing their use in nursing.

Clinical practice guidelines are "systematically developed statements to assist practitioner decisions about appropriate health care for specific clinical circumstances."1 Guidelines can be used to reduce inappropriate variations in practice and to promote the delivery of high quality, evidence-based health care. They may also provide a mechanism by which healthcare professionals can be made accountable for clinical activities. ${ }^{2}$ Although most of the development and evaluation of clinical guidelines has occurred in the field of medicine, nurses are becoming more interested in the use of guidelines as one means of facilitating evidence-based practice.

\section{Guideline development}

Clinical guidelines can be developed either locally (internal guidelines) or regionally or nationally (external guidelines). Although internal guidelines may need fewer resources and may be more likely to be adopted into clinical practice because of local ownership, ${ }^{3}$ local groups may not have the skills required for guideline development. ${ }^{5}$ An alternative is the development of guidelines at regional or national levels and subsequent modification to suit local circumstances. ${ }^{56}$

Guideline development has 4 stages. Firstly, it is essential that guidelines are based on the best available research evidence, and therefore a detailed literature search is done to identify evidence from research studies about the appropriateness and effectiveness of different clinical strategies. Next, using the research evidence, guideline construction takes place, usually through some form of small group work, with representation from as many interested parties as possible. ${ }^{7}$ Then, the guideline is tested by asking professionals not involved in the guideline development to review it for clarity, internal consistency, and acceptability. The guideline can then be tested in selected healthcare settings to see whether it is feasible for use in routine practice. Finally, the guideline should be reviewed after a specified time period and modified to take into account new knowledge.

If guidelines are to be effective, they need to have most, if not all, of the following 11 characteristics $^{8}$ : validity, cost effectiveness, reproducibility, reliability, representative development, clinical applicability, clinical flexibility, clarity, meticulous documentation, scheduled review, and unscheduled review. Validity means that if a guideline is followed it should lead to the health gains and costs predicted. This requires that the guideline be rigorously developed and consistent with available scientific evidence. Cost effectiveness means that the improvements in health care should have acceptable costs. If guidelines ignore costs and concentrate only on benefits, practices might be recommended with major implications for resource use, which are not accompanied by correspondingly large improvements in patient outcomes. Reproducibility means that given the same evidence, another guideline development group would produce similar recommendations, whereas reliability means that given the same clinical circumstances, another health professional would apply the recommendations in a similar fashion; both are more likely to occur if the guideline is developed in a systematic and rigorous manner. Guideline development should be undertaken by a group with representation from all key disciplines and interests, including patients. For a guideline to be clinically applicable, the target population should be defined in line with scientific evidence; guidelines should also be flexible by identifying exceptions and how patient preferences are to be taken into account in the decision making process. Guidelines should be clear, using precise definitions and user friendly formats. Meticulous documentation of the guideline development process should include details of who took part, methods used, and assumptions made, and should link recommendations to the available evidence, which should be graded according to its method (eg, from randomised controlled trials [grade I] to uncontrolled or consensus studies [grade III]). Guidelines should also be reviewed periodically and modified to incorporate new knowledge.

\section{Guideline dissemination and implementation}

Once a clinical guideline is ready for use, 2 stages facilitate its introduction into practice: dissemination and implementation. Dissemination refers to the method by which guidelines are made available to potential users. Implementation means ensuring that users subsequently act upon the recommendations; "implementation is a more active process, involving tailoring the message to the needs of the target audience, and actively working to overcome barriers to behaviour change." ${ }^{9}$

Dissemination strategies include publication in professional journals, sending guidelines to targeted individuals, as well as strategies involving an educational intervention. Several studies have assessed the effectiveness of different strategies: dissemination by publication or direct mailing have been found to be the least successful, ${ }^{6}{ }^{10}{ }^{11}$ but have the advantage of being cheap and reproducible. Strategies involving an educational component, especially where this is specifically targeted rather than in the form of continuing education, are more likely to result in behaviour change. ${ }^{12}$

Dissemination alone, without an appropriate implementation strategy, is unlikely to influence behaviour substantially. ${ }^{13}$ Implementation strategies try to ensure that users adopt and apply guidelines to which they have access. Grol suggests that when designing an implementation strategy, barriers to behaviour change such as structural (eg, staff workload and financial resources) and attitudinal factors (eg, acceptance of guidelines and willingness to change) should be considered. ${ }^{6}$ Appropriate interventions might be targeted at both the structure and process of care. Some implementation strategies provide accessible reminders of the guideline. For example, patient specific prompts at the time of consultation 
are thought to be a powerful strategy ${ }^{14-16}$ As well, a systematic review of randomised controlled trials found that audit and feedback had a small, but potentially worthwhile, effect on physician behaviour. ${ }^{17}$

Much of the literature on the use of clinical guidelines comes from the field of medicine. A recent systematic review for the Cochrane Collaboration, however, examined whether clinical guidelines were effective in changing the behaviour of nurses, midwives, and other health professionals. ${ }^{18}{ }^{19}$ This review identified 18 evaluations of guidelines, and all but one evaluated the introduction of guidelines targeted at nurses. Guidelines evaluated included the management of urinary catheter care, ${ }^{20}$ hypertension, ${ }^{21}$ and postoperative bleeding after cardiac surgery.2 ${ }^{22}$ Nine studies evaluated the effectiveness of guidelines compared with a no guideline control group. 4 of 5 studies that measured care processes (eg, catheter care practices and provision of dietary counselling) found significant changes in at least some of the assessed processes; 6 of 8 studies measuring patient outcomes (eg, symptom relief and length of stay) found significant differences favouring the group that received guidelines. The findings of 3 studies that evaluated different dissemination and implementation strategies suggest that educational interventions (eg, lectures or teaching sessions) are better than passive approaches (eg, postal distribution) for the dissemination of guidelines. These findings should be treated with caution because of methodological flaws: 2 studies had unit of analysis errors (ie, the unit of randomisation was the provider, but the unit of analysis was the patient). The final group of 6 studies examined whether guidelines enabled skill substitution (eg, substituting nurses for doctors in the management of low back pain) and generally found no differences between protocol based care by a nurse and physician care.

\section{Conclusions}

This review has provided some evidence that guideline based care can change nursing practice and patient outcomes. There is, however, a long way to go before guidelines meeting the 11 criteria described above are routinely used by nurses to improve patient care. Although all guidelines should be based on evidence of effectiveness, study reports of the guideline development process typically contained scant details of methods for identifying and assessing relevant evidence. Many of the guidelines identified were based on literature reviews, but there was no description of the extent to which systematic methods were used, or the criteria used to assess the quality of evidence. This calls into question the validity of the guidelines and their potential for improving patient care and outcomes.

If guidelines are to be based on evidence of effective practice, a prerequisite is high quality evidence of the benefits, harms, and costs of the targeted procedures and practices. Further research into the effectiveness of nursing practice and interventions is needed to provide this evidence base. Particular attention should be given to obtaining high quality evidence from randomised controlled trials, rather than from weaker study designs.

Nurses need to have an active role in guideline development or the adaptation of existing guidelines to local circumstances. This approach will encourage a sense of own- ership of the guideline and is more likely to lead to more positive attitudes towards it. It will also ensure that profession specific practices, and barriers and facilitating factors for bringing about behaviour change are taken into account.

When possible, the introduction of clinical guidelines should be within an evaluative framework. Nursing requires more evidence that those dissemination and implementation strategies that have proved to be most effective in changing the behaviour of physicians are also effective in changing the behaviour of nurses.

Clinical guidelines are a potential means by which evidence can be incorporated into nursing practice. More research is needed to identify the most effective ways of developing, disseminating, and implementing clinical guidelines in nursing. Only then can nurses make informed decisions about their potential for improving nursing practice and patient outcomes.

Centre for Health Services Research,

LOIS THOMAS, RN, PhD

University of Newcastle upon Tyne,

Newcastle upon Tyne, UK

1 Field MJ, Lohr KN. Clinical practice guidelines: directions for a new program. Washington, DC: National Academy Press, 1990.

2 Roval College of General Practitioners Clinical Guidelines Working Group. The development and implementation of clinical guidelines. London:
Grouling Group. The development and implementation of

3 Williamson JW. Formulating priorities for quality assurance activity. Description of a method and its application. JAMA 1978;239:631-7.

4 Putnam RW, Curry L. Impact of patient care appraisal on physician behaviour in the office setting. CMAJ 1985;132:1025-9.

5 Grol R. National standard setting for quality of care in general practice: attitudes of general practitioners and response to a set of standards. $\mathrm{Br} \mathrm{J}$ Gen Pract 1990;40:361-4.

6 Grol R. Implementing guidelines in general practice care. Quality in Health Care 1992;1:184-91.

7 Lomas J. Making clinical policy explicit. Legislative policy making and lessons for developing practice guidelines. Int J Technol Assess Health Care 1993;9:11-25.

8 Effective Health Care. Implementing clinical practice guidelines. Leeds: University of Leeds, 1994 .

9 Lomas J. Teaching old (and not so old) docs new tricks:effective ways to implement research findings, Working Paper 93-4. Toronto: McMaster University Centre research findings, Working Paper 93-4. Toronto: McMaster
for Health Economics and Policy Analysis, 1993;1-21.

10 Lomas J, Anderson GM, Domnick-Pierre K, et al. Do practice guidelines guide practice? The effect of a consensus statement on the practice of physicians. N Engl J Med 1989;321:1306-11.

11 Freemantle N, Harvey EL, Wolf F, et al. Printed educational materials to improve the behaviour of health care professionals and patient outcomes (Cochrane Review, latest version 01 Dec 1997). In: Cochrane Library. Oxford: Update Software.

12 Lomas J, Enkin M, Anderson GM, et al. Opinion leaders vs audit and feedback to implement practice guidelines. Delivery after previous cesarean section. JAMA 1991;265:2202-7.

13 Grimshaw J, Freemantle N, Wallace S, et al. Developing and implementing clinical practice guidelines. Quality in Health Care 1995;4:55-64.

14 Grimshaw JM, Russell IT. Effect of clinical guidelines on medical practice: a Grimshaw JM, Russell IT. Effect of clinical guidelines on medical practions
systematic review of rigorous evaluations. Lancet 1993;342:1317-22.

15 Emslie C, Grimshaw J, Templeton A. Do clinical guidelines improve general practice management and referral of infertile couples? BMJ 1993; 306:1728-31.

16 Lilford RJ, Kelly M, Baines A, et al. Effect of using protocols on medical care: randomised trial of three methods of taking an antenatal history. BMJ 1992;305:1181-4

17 Thomson MA, Oxman AD, Davis DA, et al. Audit and feedback to improve health professional practice and health care outcomes (Part I) (Cochrane Review, latest version 01 Dec 1997). In: Cochrane Library. Oxford: Update Software.

18 Thomas LH, McColl E, Cullum N, et al. Effect of clinical guidelines in nursing, midwifery and the therapies: a systematic review of rigorous evaluations. Quality in Health Care 1998;7:183-191.

19 Thomas LH, Cullum N, McColl E, et al. Clinical guidelines in nursing, midwifery and professions allied to medicine. (Cochrane Review, latest version 24 Nov 98.) In: Cochrane Library. Oxford: Update Software.

20 Seto WH, Ching TY, Yuen KY, et al. The enhancement of infection control in-service education by ward opinion leaders. Am J Infect Control 1991;19: in-service

21 Jewell D, Hope J. Evaluation of a nurse-run hypertension clinic in general practice. Practitioner 1988:232:484-7.

22 Zeler KM, McPharlane TJ, Salamonsen RF. Effectiveness of nursing involvement in bedside monitoring and control of coagulation status after cardiac surgery. Am J Crit Care 1992;1:70-5. 